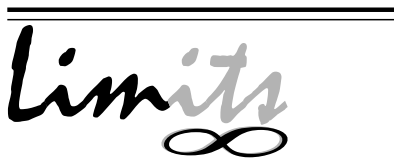

J. Math. and Its Appl.

ISSN : 1829-605X

Vol. 3, No. 1, May 2006, 27-40

\title{
Keterbatasan Operator Riesz di Ruang Morrey
}

\section{Gani Gunawan, Hendra Gunawan}

Departemen Matematika

FMIPA ITB

\begin{abstract}
Abstrak
Dengan menggunakan transformasi Fourier, didefinisikan operator $(-\Delta)^{-\frac{\alpha}{2}}, 0<\alpha<n$, yang dikenal sebagai operator Riesz atau operator integral fraksional $I_{\alpha}$, yaitu $I_{\alpha}:=(-\Delta)^{-\frac{\alpha}{2}}, 0<\alpha<n$. Dalam makalah ini akan diperlihatkan bahwa aksi dari operator tersebut bersifat terbatas dari ruang $L^{p}\left(\mathbb{R}^{n}\right)$ ke ruang $L^{q}\left(\mathbb{R}^{n}\right)$ jika dan hanya jika dengan $\frac{1}{p}-\frac{1}{q}=\frac{\alpha}{n}$ dengan $1<p<q<\infty$. Selanjutnya diperlihatkan juga bahwa operator tersebut terbatas di ruang perumumannya, khususnya di ruang Morrey.
\end{abstract}

Kata Kunci: Operator Riesz, ruang Lebesgue, ruang Morrey

\section{Pendahuluan}

Diasumsikan $f \in \mathcal{S}$, dengan $\mathcal{S}$ adalah himpunan fungsi yang terdiferensialkan tak hingga kali di $\mathbb{R}^{n}$. Transformasi Fourier dari fungsi $f$ dinotasikan dengan $f^{\wedge}$, dan didefinisikan oleh

$$
f^{\wedge}(\xi):=\int_{\mathbb{R}^{n}} f(x) e^{-2 \pi i \xi x} d x, \quad \forall \xi \in \mathbb{R}^{n}
$$

${ }^{*}$ Jur. Mat. UNISBA, Mhs S2 Jur. Mat. ITB 
Jika $\Delta f$ menotasikan Laplacian dari $f$ yang didefinisikan oleh $\Delta f:=\sum_{j=1}^{n} \frac{\partial^{2} f}{\partial x^{2}}$, maka untuk suatu $\xi \in \mathbb{R}^{n}$, berlaku $(-\Delta f)^{\wedge}(\xi)=4 \pi^{2}|\xi|^{2} f^{\wedge}(\xi)$, lihat $([5]$, halaman 308). Oleh karena itu melalui persamaan ini didefinisikan untuk $0<\alpha<n$, $\left((-\Delta)^{-\frac{\alpha}{2}} f\right)^{\wedge}(\xi)=(2 \pi|\xi|)^{-\alpha} f^{\wedge}(\xi)$, untuk setiap $\xi \in \mathbb{R}^{n}$.

Karena $\left.(-\Delta)^{-\frac{\alpha}{2}} f=\left((-\Delta)^{-\frac{\alpha}{2}} f\right)^{\wedge \vee}=\left((2 \pi|\xi|)^{-\alpha} f^{\wedge}\right)^{\vee} \operatorname{dimana}(-\Delta)^{-\frac{\alpha}{2}} f\right)^{\wedge \vee}$ adalah invers transformasi Fourier dari $\left((-\Delta)^{-\frac{\alpha}{2}} f\right)^{\wedge}$, maka untuk suatu $x \in \mathbb{R}^{n}$,

$$
\left((2 \pi|\xi|)^{-\alpha} f^{\wedge}\right)^{\vee}(x)=\frac{\Gamma\left(\frac{n-\alpha}{2}\right)}{2^{\alpha} \pi^{\frac{n}{2}} \Gamma\left(\frac{\alpha}{2}\right)} \int_{\mathbb{R}^{n}} \frac{f(y)}{|x-y|^{n-\alpha}} d y
$$

Uraian dari invers transformasi tersebut dapat dilihat pada ([5], halaman 367) atau ([7], halaman 123). Menurut Stein (lihat [2], halaman 117), dituliskan bahwa

$$
I_{\alpha} f:=(-\Delta)^{-\frac{\alpha}{2}} f, 0<\alpha<n
$$

Jadi

$$
I_{\alpha} f(x):=\frac{1}{\Gamma(\alpha)} \int_{\mathbb{R}^{n}} \frac{f(y)}{|x-y|^{n-\alpha}} d y
$$

dengan

$$
\Gamma(\alpha)=\frac{\pi^{\frac{n}{2}} 2^{\alpha} \Gamma\left(\frac{\alpha}{2}\right)}{\Gamma\left(\frac{(n-\alpha)}{2}\right)}
$$

$I_{\alpha}$ pada Persamaan 1 selanjutnya dinamakan sebagai operator Riesz atau operator integral fraksional.

Dalam makalah ini akan dilihat bagaimana aksi dari operator tersebut jika dikenakan di ruang $L^{p}=L^{p}\left(\mathbb{R}^{n}\right)$ dan ruang perumumannya, khususnya di ruang Morrey. Adapun notasi $L^{p}=L^{p}\left(\mathbb{R}^{n}\right), 1 \leq p<\infty$ disebut juga ruang Lebesgue, yaitu himpunan kelas-kelas ekuivalen fungsi sedemikian sehingga $\|f\|_{L^{p}\left(\mathbb{R}^{n}\right)}<\infty$, atau dapat ditulis $L^{p}\left(\mathbb{R}^{n}\right):=\left\{f:\|f\|_{L^{p}\left(\mathbb{R}^{n}\right)}<\infty\right\}$ dengan $\|f\|_{L^{p}\left(\mathbb{R}^{n}\right)}=\|f\|_{p}$ adalah norm dari $f$ dan didefinisikan oleh $\|f\|_{p}:=\left(\int_{\mathbb{R}^{n}}|f(x)|^{p} d x\right)^{1 / p}$, untuk setiap $x \in \mathbb{R}^{n}$. Sedangkan norm dari $f$ untuk $p=\infty$ didefinisikan oleh $\|f\|_{\infty}:=$ ess $\sup \left\{|f(x)|: x \in \mathbb{R}^{n}\right\}$ dengan ess $\sup \left\{|f(x)|: x \in \mathbb{R}^{n}\right\}$ merupakan batas atas terkecil esensial dari $|f|$.

Diawali dari suatu proposisi mengenai operator $I_{\alpha}$ di ruang $L^{p}\left(\mathbb{R}^{n}\right)$, dapat diperlihatkan bahwa melalui operator dilasi yang dikenakan pada opertor $I_{\alpha}$ diperoleh suatu syarat perlu dari keterbatasan operator $I_{\alpha}$ di ruang $L^{p}\left(\mathbb{R}^{n}\right)$. Kemudian dengan menggunakan fakta keterbatasan fungsi maksimal atau ketaksamaan Hardy-Littlewood di ruang $L^{p}\left(\mathbb{R}^{n}\right)$ dapat ditunjukan juga bahwa operator $I_{\alpha}$ merupakan operator yang terbatas dari $L^{p}\left(\mathbb{R}^{n}\right)$ ke $L^{q}\left(\mathbb{R}^{n}\right)$ asalkan $\frac{1}{p}-\frac{1}{q}=\frac{\alpha}{n}$ dengan $1<p<q<\infty$. Selanjutnya dengan menggunakan ketaksamaan Fefferman-Stein, 
dapat ditunjukan bahwa operator maksimal juga terbatas di ruang perumumannya, khususnya di ruang Morrey. Berdasar pada fakta ini, akhirnya diperoleh hasil bahwa operator $I_{\alpha}$ juga terbatas di ruang perumumannya, yakni terbatas di ruang Morrey.

\section{Keterbatasan Operator $I_{\alpha}$ di Ruang Lebesgue}

Pertama-tama akan kita lihat aksi dari operator $I_{\alpha}$ ini di ruang $L^{p}\left(\mathbb{R}^{n}\right)$ yang mempunyai sifat bahwa operator tersebut terbatas dari $L^{p}\left(\mathbb{R}^{n}\right)$ ke $L^{q}\left(\mathbb{R}^{n}\right)$ hanya jika $\frac{1}{p}-\frac{1}{q}=\frac{\alpha}{n}$. Untuk melihat sifat ini kita pandang suatu operator dilasi $\tau_{\delta}$ yang didefinisikan untuk $\delta>0$ oleh $\tau_{\delta} f(x):=f(\delta x)$. Maka dapat dinyatakan suatu lemma berikut,

\section{Lemma 2.1}

$$
\begin{array}{ll}
\text { (a) } & \left\|\tau_{\delta} f\right\|_{p}=\delta^{-n / p}\|f\|_{p} \\
\text { (b) } & \tau_{\delta^{-1}} I_{\alpha} f=\delta^{-\alpha} I_{\alpha} \tau_{\delta^{-1}} f
\end{array}
$$

\section{Bukti:}

(a).

$$
\begin{aligned}
\left\|\tau_{\delta} f\right\|_{p} & =\left(\int_{\Re^{n}}\left(\tau_{\delta} f(x)\right)^{p} d x\right)^{1 / p}=\left(\int_{\Re^{n}}(f(\delta x))^{p} d x\right)^{1 / p} \\
& =\left(\int_{\Re^{n}}\left(f\left(x^{\prime}\right)\right)^{p} \delta^{-n} d x^{\prime}\right)^{1 / p}=\delta^{-n / p}\left(\int_{\Re^{n}}\left(f\left(x^{\prime}\right)^{p} d x^{\prime}\right)^{1 / p}\right. \\
& =\delta^{-n / p}\|f\|_{p}
\end{aligned}
$$


(b).

$$
\begin{aligned}
\tau_{\delta^{-1}} I_{\alpha} f(x) & =\tau_{\delta^{-1}}\left[\frac{1}{\Gamma(\alpha)} \int_{\Re^{n}}|x-y|^{-n+\alpha} f(y) d y\right] \\
& =\frac{1}{\Gamma(\alpha)} \int_{\Re^{n}}\left|\frac{x}{\delta}-y\right|^{-n+\alpha} f(y) d y \\
& =\frac{1}{\Gamma(\alpha)} \int_{\Re^{n}}|x-\delta y|^{-n+\alpha} \delta^{n-\alpha} f(y) d y \\
& =\frac{1}{\Gamma(\alpha)} \int_{\Re^{n}}\left|x-y^{\prime}\right|^{-n+\alpha} \delta^{n} \delta^{-\alpha} f\left(\frac{y^{\prime}}{\delta}\right) d y \\
& =\delta^{-\alpha} \frac{1}{\Gamma(\alpha)} \int_{\Re^{n}}\left|x-y^{\prime}\right|^{-n+\alpha} f\left(\frac{y^{\prime}}{\delta}\right) d y^{\prime} \\
& =\delta^{-\alpha} \frac{1}{\Gamma(\alpha)} \int_{\Re^{n}}\left|x-y^{\prime}\right|^{-n+\alpha} \tau_{\delta^{-1}} f\left(y^{\prime}\right) d y^{\prime} \\
& =\delta^{-\alpha} I_{\alpha} \tau_{\delta^{-1}} f(x)
\end{aligned}
$$

Akibat dari Lemma 2.1 tersebut diperoleh syarat perlu keterbatasan fungsi maksimal di ruang $L^{p}\left(\mathbb{R}^{n}\right)$ yang dinyatakan dalam proposisi berikut.

Proposisi 2.2 Jika ketaksamaan

$$
\left\|I_{\alpha} f\right\|_{q} \leq A\|f\|_{p}, \quad 0<\alpha<n
$$

dipenuhi untuk setiap $f$ dan untuk suatu konstanta A, maka $\frac{1}{p}-\frac{1}{q}=\frac{\alpha}{n}$.

\section{Bukti:}

Akibat dari Lemma 2.1 bagian (a), dapat ditulis $\left\|\tau_{\delta^{-1}} I_{\alpha} f\right\|_{q}=\delta^{n / q}\left\|I_{\alpha} f\right\|_{q}$ bahwa sehingga diperoleh

$$
\left\|I_{\alpha} f\right\|_{q}=\delta^{-\frac{n}{q}}\left\|\tau_{\delta^{-1}} I_{\alpha} f\right\|_{q}
$$

Menurut Lemma 2.1 bagian (b), $\tau_{\delta^{-1}} I_{\alpha} f(x)=\delta^{-\alpha} I_{\alpha} \tau_{\delta^{-1}} f(x)$, maka diperoleh

$$
\left\|I_{\alpha} f\right\|_{q}=\delta^{-\frac{n}{q}}\left\|\tau_{\delta^{-1}} I_{\alpha} f\right\|_{q}=\delta^{-\frac{n}{q}}\left\|\delta^{-\alpha} I_{\alpha} \tau_{\delta^{-1}} f\right\|_{q}=\delta^{-\frac{n}{q}-\alpha}\left\|I_{\alpha} \tau_{\delta^{-1}} f\right\|_{q}
$$

Karena Ketaksamaan 2 dipenuhi, maka diperoleh untuk suatu konstanta $A$ (dalam hal ini $A$ dapat diasumsikan sebagai konstanta terkecil yang memenuhi 2)

$$
\left\|I_{\alpha} f\right\|_{q} \leq A \delta^{-\frac{n}{q}-\alpha}\left\|\tau_{\delta^{-1}} f\right\|_{q}
$$

Menurut Lemma 2.1 bagian (a), $\left\|\tau_{\delta^{-1}} f\right\|_{q}=\delta^{\frac{n}{q}}\|f\|_{q}$. Jadi Ketaksamaan 3 dapat ditulis

$$
\left\|I_{\alpha} f\right\|_{q} \leq A \delta^{-\frac{n}{p}-\frac{n}{q}-\alpha}\|f\|_{q}
$$


Ini mungkin hanya jika $\frac{n}{p}-\frac{n}{q}-\alpha=0$.

Diamati lebih lanjut, syarat cukup untuk proposisi tersebut juga dapat dipenuhi. Namun untuk kasus $p=1,\left(\right.$ maka $\left.q=\frac{n}{n-\alpha}\right)$ dan $q=8$, (maka $\left.p=\frac{n}{\alpha}\right)$ gagal untuk dapat dipenuhi, lihat ([2], halaman 119). Oleh karena itu, setelah melalui pengamatan ini dapat diformulasikan teorema positifnya, yang disebut teorema Hardy-Littlewood-Sobolev. Persisnya kita mempunyai suatu teorema berikut.

Teorema 2.3 (Hardy-Littlewood-Sobolev) Jika $\frac{1}{p}=\frac{1}{q}-\frac{\alpha}{n}$ dan $1<p<q<\infty$ dengan $0<\alpha<n$, maka

$$
\left\|f^{*}|\cdot|^{\alpha-n}\right\|_{L^{q}\left(\mathbb{R}^{n}\right)} \leq A_{p, q}\|f\|_{L^{q}\left(\mathbb{R}^{n}\right)}
$$

Sebelum membuktikan Teorema 2.3 tersebut, pertama-tama perhatikan beberapa definisi, sifat dan fakta yang ada sebagai konsep yang mendasarinya. Sebelumnya, didefinisikan fungsi maksimal Hardy-Littlewood,

$$
M f(x):=\sup _{0<R<\infty} \frac{1}{\mu(B(x, R))} \int_{B(x, R)}|f(y)| d y, \quad \forall x \in \mathbb{R}^{n}
$$

dengan $f \in L_{\text {loc }}^{1}\left(\mathbb{R}^{n}\right)$, yaitu fungsi terintegralkan secara lokal di $\mathbb{R}^{n}$, dan $\mu(B(x, R))$ adalah ukuran Lebesgue $B(x, R)$ di $\mathbb{R}^{n}$. Dalam hal ini $B(R)=B(x, R)$ adalah bola buka di $\mathbb{R}^{n}$ yang berpusat di titik $x \in \mathbb{R}^{n}$ dengan radius $R>0$, yaitu $B(x, R):=\left\{y \in \mathbb{R}^{n}:|x-y|<R\right\}$, untuk setiap $x \in \mathbb{R}^{n}$ dan $R>0$. Selanjutnya teorema Hardy-Littlewood berikut, yang menyatakan bahwa operator maksimal M tersebut terbatas di ruang $L^{p}\left(\mathbb{R}^{n}\right)$, lihat [8].

Teorema 2.4 (Hardy-Littlewood) Jika $1<p<\infty$ dan $f \in L^{p}$, maka $M f \in L^{p}$ dan $\|M f\|_{p} \leq C_{p, n}\|f\|_{p}$.

Definisi 2.5 Fungsi $f$ di $\mathbb{R}^{n}$ disebut fungsi radial jika nilai $f(x)$ hanya bergantung pada $|x|$ untuk $x \in \mathbb{R}^{n}$.

Lemma 2.6 Misalkan $\psi$ fungsi non negatif di $\mathbb{R}^{n}$. Jika $\psi$ fungsi radial yang turun, maka

$$
\sup \left\{\left|f^{*} \psi_{t}(x)\right|: t>0\right\} \leq M f(x) \int_{\mathbb{R}^{n}} \psi d y
$$

dengan Mf merupakan fungsi maksimal dan $\psi_{t}=t^{-n} \psi\left(\frac{x}{t}\right)$ 


\section{Bukti:}

Lihat ([3], halaman 57)

Lemma 2.7 Jika $f$ fungsi radial, maka

$$
\int_{\mathbb{R}^{n}} f(x) d x=\omega_{n-1} \int_{0}^{\infty} f(r) r^{n-1} d r, \quad x \in \mathbb{R}^{n}
$$

dengan $\omega_{n-1}=\frac{2 \pi^{n / 2}}{\Gamma\left(\frac{n}{2}\right)}$

Lihat ([5], halaman 407).

Lemma 2.8 Misalkan $\phi(y)=|y|^{-k} \chi_{B(R)}(y), y \in \mathbb{R}^{n}$ dan $y \neq 0$. Maka untuk setiap $R>0$ dan $0<k<n$,

(i). $\phi$ adalah fungsi turun secara radial

(ii). $\phi$ terintegralkan

\section{Bukti:}

(i). Ambil $y_{1}, y_{2}$ dengan $0<\left|y_{1}\right|<\left|y_{2}\right| \leq R$, maka $\left|y_{1}\right|^{-k}>\left|y_{2}\right|^{-k}$. Karena $\chi_{B(R)}(y)=1$ untuk setiap $y \in B(R)$, sehingga diperoleh $\chi_{B(R)}\left(y_{1}\right)=$ $\chi_{B(R)}\left(y_{2}\right)=1$. Jadi $\phi\left(y_{1}\right)=\left|y_{1}\right|^{-k} \chi_{B(R)}\left(y_{1}\right) \geq\left|y_{2}\right|^{-k} \chi_{B(R)}\left(y_{2}\right)=\phi\left(y_{2}\right)$. Ini berarti $\phi(y)$ adalah fungsi turun secara radial untuk setiap $y \in \mathbb{R}^{n}$.

(ii).

$$
\begin{aligned}
\int_{\mathbb{R}^{n}} \phi(y) d y & =\int_{\mathbb{R}^{n}}|y|^{-k} \chi_{B(R)}(y) d y=\int_{|y|<R}|y|^{-k} d y \\
& =\int_{\theta \in S^{n-1}}\left(\int_{0}^{R}|r|^{-k} r^{n-1} d r\right) d \theta \\
& =\int_{\theta \in S^{n-1}}\left(\frac{1}{n-k}|r|^{n-k}\right)_{r=0}^{r=R} d \theta \\
& =\int_{\theta \in S^{n-1}} \frac{R^{n-k}}{n-k} d \theta=\frac{R^{n-k}}{n-k} \int_{\theta \in S^{n-1}} d \theta \\
& =\frac{R^{n-k}}{n-k} \omega_{n-1}=\frac{R^{n-k}}{n-k} \frac{2 \pi^{n / 2}}{\Gamma\left(\frac{n}{2}\right)}<\infty
\end{aligned}
$$


Lemma 2.9 Misalkan $\psi(y)=|y|^{-k} \chi_{C_{B(R)}}(y)$ dengan $y \in \mathbb{R}^{n}, y \neq 0$ dan $R>0$. Jika $0<k<n$ dan $n-k p^{\prime}<0$, maka $\psi \in L^{p^{\prime}}$

Bukti

$$
\begin{aligned}
\int_{\Re^{n}}|\psi(y)|^{p^{\prime}} d y & =\left.\left.\int_{\Re^{n}}|| y\right|^{-k} \chi_{C_{B(R)}}(y)\right|^{p^{\prime}} d y \\
& =\int_{\Re^{n}}|y|^{-k p^{\prime}} \chi_{C_{B(R)}}(y) d y \\
& =\int_{|y| \geq \Re}|y|^{-k p^{\prime}} d y \\
& =\int_{\theta \in s^{n-1}}\left(\int_{\Re}^{\infty}|r|^{-k p^{\prime}} r^{n-1} d r\right) d \theta \\
& =\int_{\theta \in s^{n-1}}\left(\frac{1}{n-k p^{\prime}} r^{n-k p^{\prime}}\right)_{r=\Re}^{r=\infty} d \theta, n-k p^{\prime}<0 \\
& =\frac{R^{n-k p^{\prime}}}{k p^{\prime}-n} \int_{\theta \in s^{n-1}} d \theta=\frac{R^{n-k p^{\prime}}}{k p^{\prime}-n} \omega_{n-1} \\
& =\frac{R^{n-k p^{\prime}}}{k p^{\prime}-n} \frac{2 \pi^{n / 2}}{\Gamma(n / 2)}<\infty
\end{aligned}
$$

Jadi $\psi \in L^{p^{\prime}}$.

Selanjutnya kita lihat pembuktian Teorema 2.3 sebagai berikut, Untuk $R>0$ dapat dituliskan bahwa

$$
\begin{aligned}
\left(f^{*}|\cdot|^{\alpha-n}\right)(x) & =\int_{\mathbb{R}^{n}} f(x-y)|y|^{\alpha-n} d y \\
& =\int_{|y|<R} f(x-y)|y|^{\alpha-n} d y+\int_{|y| \geq R} f(x-y)|y|^{\alpha-n} d y
\end{aligned}
$$

Perhatikan suku pertama Persamaan 5, dapat ditulis menjadi

$$
\int_{|y|<R} f(x-y)|y|^{\alpha-n} d y=\left(f^{*}|\cdot|^{\alpha-n} \chi_{B(R)}(y)\right)(x)
$$

dengan

$$
\chi_{B(R)}(y)=\left\{\begin{array}{lll}
1, & \text { jika } & y \in B(R) \\
0, & \text { jika } & y \notin B(R)
\end{array}\right.
$$

Dan menurut Definisi 2.5, jika dimisalkan $\phi(y)=|y|^{\alpha-n} \chi_{B(R)}(y)$ maka $\phi$ merupakan fungsi radial. Berdasarkan Lemma 2.8 diperoleh bahwa $\phi$ adalah fungsi 
turun yang terintegralkan. Akibatnya menurut Lemma 2.6, jika $\phi_{t}=t^{-n} \phi(x / t)$, maka

$$
\begin{aligned}
\left|f^{*} \phi(x)\right|=\left|f^{*} \phi_{1}(x)\right| & \leq \sup \left\{\left|f^{*} \phi_{t}(x)\right|: t>0\right\} \\
& \leq M f(x) \int_{\mathbb{R}^{n}} \phi(y) d y \\
& =C_{1} R^{\alpha} M f(x),(\phi \text { terintegralkan })
\end{aligned}
$$

untuk suatu bilangan real $C_{1}$.

Suku kedua dari Persamaan 5 dapat dituliskan menjadi

$\int_{|y| \geq R} f(x-y)|y|^{\alpha-n} d y=\left(f^{*}|\cdot|^{\alpha-n} \chi_{C_{B(R)}}(y)\right)(x)=\int f(x-y)|y|^{\alpha-n} \chi_{C_{B(R)}}(y) d y$ dengan

$$
\chi_{C_{B(R)}}(y)=\left\{\begin{array}{lll}
1, & \text { jika } & y \in C_{B(R)} \\
0, & \text { jika } & y \notin C_{B(R)}
\end{array}\right.
$$

Oleh karena itu dapat ditulis

$$
\begin{aligned}
\left.\left|\int_{|y| \geq \Re} f(x-y)\right| y\right|^{\alpha-n} d y \mid & =\left.\left|\int f(x-y)\right| y\right|^{\alpha-n} \chi_{C_{B(R)}}(y) d y \mid \\
& \leq\left.\int|f(x-y)| y\right|^{\alpha-n} \chi_{C_{B(R)}}(y) \mid d y
\end{aligned}
$$

Misalkan $u=-y$, maka dapat ditulis

$$
\int f(x-y)|y|^{\alpha-n} \chi_{C_{B(R)}}(y) d y=\int f(u+x)|u|^{\alpha-n} \chi_{C_{B(R)}}(u) d u .
$$

Jadi menurut ketaksamaan Holder diperoleh

$$
\begin{aligned}
\left.\left|\int_{|y| \geq R} f(x-y)\right| y\right|^{\alpha-n} d y \mid & \leq\left.\int|f(u+x)| u\right|^{\alpha-n} \chi_{C_{B(R)}}(u) \mid d u \\
& =\left\|f_{+x}|u|^{\alpha-n} \chi_{C_{B(R)}}(u)\right\|_{1} \\
& \leq\left.\left\|f_{+x}\right\|\left\|_{p}\right\| u\right|^{\alpha-n} \chi_{C_{B(R)}}(u) \|_{p^{\prime}} \\
& =\|f\|_{p}\left\||y|^{\alpha-n} \chi_{C_{B(R)}}(y)\right\|_{p^{\prime}} \\
& =\|f\|_{p}\|\psi\|_{p^{\prime}}
\end{aligned}
$$

dengan $\psi=|\cdot|{ }^{\alpha-n} \chi_{C_{B(R)}}$.

Perhatikan Lemma 2.9 jika $k=n-\alpha$, maka

$$
k p^{\prime}-n=\left(\frac{n}{q}-\frac{n}{p}+n\right) p^{\prime}-n=n\left(\frac{p^{\prime}}{q}\right)>0 .
$$


Akibatnya diperoleh $n-k p^{\prime}<0$. Sehingga menurut Lemma 2.9, dapat dikatakan bahwa $\psi \in L^{p^{\prime}}$. Oleh karenanya dapat dipandang untuk suatu bilangan real $C_{2}$

$$
\|\psi\|_{p^{\prime}}=C_{2} R^{-n / q}
$$

Dengan demikian Ketaksamaan 7 menjadi

$$
\left.\left|\int_{|y| \geq R} f(x-y)\right| y\right|^{\alpha-n} d y \mid \leq C_{2} R^{-n / q}\|f\|_{p}
$$

untuk suatu bilangan real $C_{2}$. Dari Persamaan 5, 6 dan 9 diperoleh

$$
\left|\left(f^{*}|\cdot|^{\alpha-n}\right)(x)\right| \leq C_{1} R^{\alpha} M f(x)+C_{2}\|f\|_{p} R^{-n / q} \leq A\left[R^{\alpha} M f(x)+\|f\|_{p} R^{-n / q}\right]
$$

dimana $A=\max \left\{C_{1}, C_{2}\right\}$.

Selanjutnya pilih $R$ sehingga $R^{\alpha} M f(x)=\|f\|_{p} R^{-n / q}$ atau $R^{-n / p}=\frac{M f(x)}{\|f\|_{p}}$. Jadi jika dipilih $R^{-n / p}=\frac{M f(x)}{\|f\|_{p}}$, maka

$$
\left|\left(f^{*}|\cdot|^{\alpha-n}\right)(x)\right| \leq A[M f(x)]^{p / q}\|f\|_{p}^{1-p / q}
$$

Sehingga diperoleh

$$
\begin{aligned}
\left\|f^{*} \mid \cdot\right\|^{\alpha-n} \|_{q} & =\left(\int\left|\left(f^{*}|\cdot|^{\alpha-n}\right)(x)\right|^{q} d x\right)^{1 / q} \\
& \leq\left[\int\left(A[M f(x)]^{p / q}\|f\|_{p}^{1-p / q}\right)^{q} d x\right]^{1 / q} \\
& \leq A\|f\|_{p}^{1-p / q}\left[\int(M f(x))^{p}\right]^{1 / q} \\
& \leq A\|f\|_{p}^{1-p / q}\|M f\|_{p}^{p / q} \\
& \leq A\|f\|_{p}^{1-p / q}\|f\|_{p}^{p / q}=C\|f\|_{p}
\end{aligned}
$$

untuk suatu konstanta $C$ yang tergantung pada $p, q$.

Dengan demikian dapat disimpulkan bahwa

$$
\left\|f^{*} \mid \cdot\right\|^{\alpha-n}\left\|_{L^{q}\left(\mathbb{R}^{n}\right)} \leq A_{p, q}\right\| f \|_{L^{q}\left(\mathbb{R}^{n}\right)}
$$

\section{Keterbatasan Operator I di Ruang Morrey}

Ruang Morrey $L^{p, \lambda}\left(\mathbb{R}^{n}\right)$ pertama kali diperkenalkan oleh C.B. Morrey pada tahun 1938 dalam suatu jurnal matematika dengan judul on the solutions of quasi linear elliptic partial differential equations. Selanjutnya ruang Morrey banyak ditemukan 
pada saat mempelajari perilaku operator Schrodinger dan teori potensial. Tujuan utama kita dalam pembahasan pada bagian ini, akan menunjukan bahwa operator $I_{\alpha}$ juga terbatas di ruang Morrey $L^{p, \lambda}\left(\mathbb{R}^{n}\right)$ ke $L^{q, \lambda}\left(\mathbb{R}^{n}\right)$ asalkan $\frac{1}{p}-\frac{1}{q}=\frac{\alpha}{n-\lambda}$ dan $0 \leq \lambda<n-\alpha p$. Adapun ruang Morrey itu sendiri didefinisikan sebagai himpunan semua fungsi $f$ yang terintegralkan secara lokal pada $\mathbb{R}^{n}$, yaitu seperti yang dinyatakan dalam definisi berikut,

Definisi 3.1 Untuk $1 \leq p<\infty$ dan $0 \leq \lambda \leq n$, ruang Morrey $L^{p, \lambda}\left(\mathbb{R}^{n}\right)$ didefinisikan oleh

$$
L^{p, \lambda}\left(\mathbb{R}^{n}\right):=\left\{f \in L_{l o c}^{p}\left(\mathbb{R}^{n}\right):\|f\|_{L^{p, \lambda}\left(\mathbb{R}^{n}\right)}<+\infty\right\}
$$

dengan

$$
\|f\|_{L^{p, \lambda}}\left(\mathbb{R}^{n}\right):=\sup _{B=B(x, R)}\left(\frac{1}{R^{\lambda}} \int_{B}|f(x)|^{p} d x\right)^{1 / p}
$$

Dalam hal ini notasi $B=B(x, R)$ merupakan bola buka berdimensi $n$ dengan pusat $x \in R^{n}$ dan berjari-jari $R>0$, yaitu $B(x, R):=\left\{y \in \Re^{n}:|x-y|<R\right\}$. Untuk setiap bola $B=B(x, R)$ berdimensi n ini dipunyai suatu fakta bahwa

$$
|B| \leq C R^{n}
$$

dimana $n$ adalah suatu bilangan dimensi yang tetap dan $C$ adalah konstanta yang tidak bergantung pada $x$ dan $R$, dengan $|B|$ menyatakan ukuran Lebesgue dari $B$, lihat ([5], halaman 3). Dari fakta 10 tersebut dapat dinyatakan suatu lemma berikut.

Lemma 3.2 Untuk setiap $\alpha>0$, maka berlaku $\int_{B(x, R)} \frac{1}{|x-y|^{n-\alpha}} d y \leq C R^{\alpha}$ untuk suatu konstanta real $C$.

\section{Bukti:}

Jika $n \leq \alpha$, maka jelas bahwa menurut fakta $(*), \int_{B(x, R)} \frac{1}{|x-y|^{n-\alpha}} d y=|B| \leq$ $C R^{\alpha}$. Sekarang jika $n<\alpha$, maka

$$
\begin{aligned}
\int_{B(x, R)} \frac{1}{|x-y|^{n-\alpha}} d y & \leq \sum_{j=0_{2^{-j-1}}}^{\infty} \int_{R \leq|x-y|<2^{-j} R} \frac{1}{|x-y|^{n-\alpha}} d y \\
& \leq \sum_{j=0}^{\infty} \frac{1}{\left(2^{-j-1} R\right)^{n-\alpha}}\left|B\left(x, 2^{-j} R\right)\right| \\
& \leq \sum_{j=0}^{\infty} \frac{\left(2^{(j+1)(n-\alpha)}\right.}{R^{n-\alpha}} C\left(2^{-j} R\right)^{n} \\
& =C \sum_{j=0}^{\infty} 2^{-\alpha j} R^{\alpha}=C R^{\alpha}
\end{aligned}
$$


Selanjutnya sebelum diperlihatkan suatu fakta yang menyatakan bahwa operator $I_{\alpha}$ juga terbatas di ruang Morrey, yakni dari $L^{p, \lambda}\left(\mathbb{R}^{n}\right)$, ke $L^{q, \lambda}\left(\mathbb{R}^{n}\right)$, terlebih dahulu disajikan suatu ketaksamaan Fefferman-Stein yang buktinya dapat dilihat di ([3], halaman 53).

Teorema 3.3 (Fefferman-Stein) Misalkan $\omega$ adalah fungsi tak negatif dan $f$ adalah fungsi terintegralkan secara lokal di $\mathbb{R}^{n}$. Maka terdapat $C_{p}>0$ sedemikian sehingga

$$
\int_{\mathbb{R}^{n}}|M f(x)|^{p} \omega(x) d x \leq C_{p} \int_{\mathbb{R}^{n}}|f(x)|^{p} M \omega(x) d x
$$

Dengan menggunakan ketaksamaan Fefferman-Stein ini diperoleh suatu fakta bahwa operator maksimal juga terbatas di ruang Morrey. Seperti dinyatakan dalam teorema berikut,

Teorema 3.4 (Chiarenza-Frasca) Misal $1<p<\infty$ dan $0 \leq \lambda<n$. Maka $\|M f\|_{L^{p, \lambda}} \leq C\|f\|_{L^{p, \lambda}}$, untuk suatu konstanta $C$ yang tidak bergantung pada $f$.

\section{Bukti:}

Menurut ketaksamaan yang dinyatakan dalam Teorema 3.3, maka

$$
\int_{\mathbb{R}^{n}}|M f(x)|^{p} \chi(x) d x \leq C \int_{\mathbb{R}^{n}}|f(x)|^{p}(M \chi(x)) d x
$$

untuk suatu fungsi $f$ dan $\chi$ yang tak negatif. Ambil $f \in L^{p, \lambda}$, dan $\chi$ adalah fungsi karakteristik pada bola $B_{R}=B\left(x_{0}, R\right)$. Maka menurut Ketaksamaan 11 di atas diperoleh

$$
\int_{B_{R}}|M f(x)|^{p} d x \leq C\|f\|_{L^{p, \lambda}}^{p}\left\{(2 R)^{\lambda}+\sum_{k=1}^{\infty} \frac{1}{\left(2^{k-1} R\right)^{n}}\left(2^{k+1} R\right)^{\lambda}\right\} \subseteq C\|f\|_{L^{p, \lambda}}^{p} R^{\lambda}
$$

Jadi didapat

$$
\frac{1}{R^{\frac{\lambda}{p}}}\left(\int_{B_{R}}|M f(x)|^{p}\right)^{\frac{1}{p}} \leq C\|f\|_{L^{p, \lambda}}^{p}
$$

untuk suatu konstanta $\mathrm{C}$

Berdasarkan pada fakta keterbatasan fungsi maksimal $M f$ di ruang Morrey, maka dapat dibuktikan suatu pernyataan teorema berikut, bahwa operator integral fraksional $I_{\alpha}$ juga terbatas di ruang Morrey.

Teorema 3.5 (Chiarenza-Frasca) Jika $\frac{1}{q}=\frac{1}{p}-\frac{\alpha}{n-\lambda}, \quad 0<\alpha<n, \quad 1<p<\frac{n}{\alpha}$ dan $0 \leq \lambda<n-\alpha p$. Maka terdapat $C_{p, q}>0$ sedemikian sehingga

$$
\left\|I_{\alpha} f\right\|_{L^{q, \lambda}} \leq C_{p, q}\|f\|_{L^{q, \lambda}}
$$




\section{Bukti:}

Misalkan $f \in L^{p, \lambda}$, dan $f \neq 0$, maka untuk $x \in \mathbb{R}^{n}$ dan $r>0$ tuliskan $I_{\alpha} f(x):=$ $I_{1}(x)+I_{2}(x)$, dengan

$$
I_{1}(x):=\int_{|x-y|<R} \frac{f(y)}{|x-y|^{n-\alpha}} d y, \quad \text { dan } \quad I_{2}(x):=\int_{|x-y| \geq R} \frac{f(y)}{|x-y|^{n-\alpha}} d y
$$

Untuk $I_{1}(x)$ diperoleh

$$
\left|I_{1}(x)\right| \leq \int_{|x-y|<R} \frac{|f(y)|}{|x-y|^{n-\alpha}} d y \leq C \sum_{k=-\infty}^{-1}\left(2^{k} R\right)^{\alpha} M f(x) d y \leq C R^{\alpha} M f(x)
$$

Sementara itu, untuk $I_{2}(x)$ diperoleh

$$
\left|I_{2}(x)\right| \leq \int_{|x-y| \geq R} \frac{|f(y)|}{|x-y|^{n-\alpha}} d y \leq C R^{\alpha-\frac{n}{p}+\frac{\lambda}{p}}\|f\|_{p, \lambda} \leq C R^{\alpha+\frac{\lambda-n}{p}}\|f\|_{L^{p, \lambda}}
$$

Sehingga untuk setiap $x \in \mathbb{R}^{n}$ dan $R>0$ diperoleh

$$
\left|I_{\alpha} f(x)\right| \leq C\left(R^{\alpha} M f(x)+R^{\alpha+\frac{\lambda-n}{p}}\|f\|_{L^{p, \lambda}}\right)
$$

Selanjutnya dengan memilih $R_{0}=\left(\frac{M f(x)}{\|f\|_{L^{p, \lambda}}}\right)^{\frac{p}{n-\lambda}}>0$ dan mensubstitusikannya ke dalam Ketaksamaan 12, sehingga didapat

$$
\left|I_{\alpha} f(x)\right| \leq C(M f(x))^{\frac{n-\lambda-p \alpha}{n-\lambda}}\|f\|_{L p, \lambda}^{\frac{p \alpha}{n-\lambda}} \leq C(M f(x))^{\frac{p}{q}}\|f\|_{L^{p, \lambda}}^{\frac{q-p}{q}}
$$

Dengan menggunakan fakta bahwa fungsi maksimal terbatas di $L^{p, \lambda}$, maka diperoleh ketaksamaan

$$
\left\|I_{\alpha} f\right\|_{L^{q, \lambda}} \leq C\|f\|_{L^{p, \lambda}}
$$

untuk suatu konstanta $C$ yang hanya tergantung pada $p$ dan $q$.

Perhatikan bahwa jika $\lambda=0$ maka $L^{p, 0}\left(\mathbb{R}^{n}\right)=L^{p}\left(\mathbb{R}^{n}\right)$. Ini berarti akan diperoleh kembali keterbatasan $I_{\alpha}$ dari $L^{p}\left(\mathbb{R}^{n}\right)$ ke $L^{q}\left(\mathbb{R}^{n}\right)$. Sedangkan jika $\lambda=n$, maka diperoleh $L^{p, \lambda}\left(\mathbb{R}^{n}\right)=L^{\infty}\left(\mathbb{R}^{n}\right)$. Dalam hal $\lambda=n$ ini, keterbatasan operator $I_{\alpha}$ dari $L^{p}\left(\mathbb{R}^{n}\right)$ ke $L^{q}\left(\mathbb{R}^{n}\right)$ menjadi tidak berlaku, seperti yang ditunjukan dalam ([2], halaman 119). 


\section{Kesimpulan}

Melalui operator dilasi yang dikenakan terhadap operator integral fraksional, dan dengan adanya fakta keterbatasan fungsi maksimal di ruang $L^{p}\left(\mathbb{R}^{n}\right)$, diperoleh fakta bahwa untuk $0<\alpha<n$, operator integral fraksional $I_{\alpha}$ yang didefinisikan sebagai

$$
I_{\alpha} f(x):=\frac{1}{\Gamma(\alpha)} \int_{R^{n}} \frac{f(y)}{|x-y|^{n-\alpha}} d y
$$

merupakan operator terbatas dari $L^{p}\left(\mathbb{R}^{n}\right)$ ke $L^{q}\left(\mathbb{R}^{n}\right)$ dengan $\frac{1}{p}-\frac{1}{q}=\frac{\alpha}{n}$ dan $1<p<q<\infty$. Persisnya kita mempunyai ketaksamaan

$$
\left\|I_{\alpha} f\right\| \leq C_{p, q}\|f\|_{p}
$$

jika dan hanya jika $\frac{1}{p}-\frac{1}{q}=\frac{\alpha}{n}$ dan $1<p<q<\infty$.

Selanjutnya di ruang perumumannya, khususnya di ruang Morrey $L^{p, \lambda},\left(\mathbb{R}^{n}\right)$, dengan menggunakan ketaksamaan Fefferman-Stein diperoleh fakta bahwa operator maksimal juga terbatas di ruang perumumannya. Akibatnya dengan memanfaatkan fakta ini diperoleh hasil bahwa operator integral fraksional $I_{\alpha}$ juga terbatas di ruang perumumannya, yaitu $I_{\alpha}$ terbatas dari ruang $L^{p, \lambda}\left(\mathbb{R}^{n}\right)$ ke $L^{q, \lambda}\left(\mathbb{R}^{n}\right)$ dengan $\frac{1}{p}-\frac{1}{q}=\frac{\alpha}{n-\alpha}$ dan $0 \leq \lambda<n-\alpha p$.

\section{Ucapan Terima Kasih.}

Kedua penulis berterima kasih kepada LPPM-ITB untuk dana Riset ITB Tahun 2006 No. 0004/K01.03.2/PL2.1.5/I/2006.

\section{Pustaka}

[1] E. Nakai, Recent topics of fractional integrals, Departemen of Mathematics, Osaka Kyoiku University Kashiwara, Osaka 582-8582, Japan.

[2] E.M. Stein, Singular integrals and differentiability properties of functions, Princenton University Press, Princenton, N.J, 1970.

[3] E.M. Stein, Harmonic Analysis: real variable methods, orthogonality, and oscillatory integrals, Princenton University Press, Princenton, New Jersey, 1993.

[4] F. Chiarenza and M.Frasca, Morrey spaces and Hardy Littlewood maximal function, rend. Mat. 7, 273-279, 1987. 
[5] G.B. Folland, Fourier analysis and its applications, Wadsworth \& Brooks/Cole, Pacific Grove, CA, 1992.

[6] J.G. Cuerva and A.E. Gatto, Boundedness properties of fractional integral operators associated to non doubling measures, Mathematics Subject Classification, DePaul University, Spain, 1991.

[7] M. Loss and H. Elliot, Analysis, Graduate student in mathematics, volume 4, 2001.

[8] R. Fefferman, Maximal functions in analysis, The University of Chicago REU, 2005. 